\title{
Evaluation of the Decision Support Systems
}

\author{
Jallal Manar, Bouhaji Mouna, Ait Moudden Naima, Housbane Samy, Serhier Zineb and Bennani Othmani \\ Mohammed \\ Medical Informatics Laboratory, Faculty of Medicine and Pharmacy of Casablanca, Ibn Rochd University Hospital Center, \\ Casablanca 96353, Morocco
}

\begin{abstract}
MDSSs (medical decision support systems) are computer applications providing clinicians with suitable information about a patient's clinical situation, as well as knowledge relevant to the situation, appropriately filtered and presented, to improve the quality of care and the patients' health. As is the case with any intervention aiming at influencing medical practices and their impact on the patients' health, it seems appropriate to question their real value through a methodology for reliable evaluation. Evaluation methods are necessarily dependent on the questions raised and it is common for different questions to lead to different methodologies and different processes of data collection and analysis. In the light of this, better consideration and better implementation of the evaluation processes of these systems are major criteria on which their future depends.
\end{abstract}

Key words: DSSs (decision support systems), clinical, health care quality, evaluation.

\section{Introduction}

Over the years, and thanks to advances in imaging, the development of biological tests, and the steady introduction of new medications or other therapeutic procedures on the market, medical knowledge has developed. However, the human memory is limited and the practitioner cannot memorize all the knowledge he needs for his daily practice. As a result, patient care, while complying with good clinical practice as outlined in guides distributed by national agencies, requires the practitioner to be equipped to carry out these complex tasks. This is the aim of computerized MDSSs (medical decision support systems), the implementation of which is essential as many studies have published numerous errors in the management of patients every year.

Decision-making systems aim to assist man by replacing or reproducing human reasoning which simultaneously implies the notions of knowledge, uncertainty, experience and risk to that every practitioner encounters in his daily practice [1].

DSSs (decision support systems) are generally

Corresponding author: JALLAL Manar, Ph.D., research fields: medical computing. designed to integrate a medical knowledge base, patient data and an inference engine to generate specific counseling for the situations faced [2]. As soon as they appeared in the 1980s, DSSs in medicine proved their usefulness and success in different areas that have motivated their development. As in any intervention seeking to influence medical practices and their impact on the patients' health, they must be evaluated. However, the scope of the questions that can be raised for the evaluation of these systems is considerable. In this respect, many evaluation studies are present in the literature. The authors often seek to evaluate the impact on quality of care through indicators they do not always agree on. Evaluation would then not only measure and explore the properties of a DSS in its design, implementation and implementation aspects, but also the art of measuring quality, cost, effects and impacts of these systems in the health care environment to establish an evidence-based practice for their use in medical practice [3].

The aim of our work is to describe the methodology for evaluating DSSs taking into account the clinical effects, the impact on professional practices, the 
knowledge represented, the technical requirements, the users and the level of interoperability.

\section{Evaluation Areas of DSSs}

The introduction of a DSS is not limited to the specification of needs, the acquisition and deployment of the system; it is a complex intervention that can lead to radical changes in the work process of practitioners and caregivers and can significantly change the way care is delivered. Therefore, proposing a unique methodology valid for any need for evaluation of DSSs is difficult. As such, the objectives of the evaluation may differ depending on the stage of development or the specific purposes of the systems.

The areas of evaluation of DSSs can be divided into several categories, namely [4]: the impact on quality and safety of care, the impact on clinical work processes and care organization, the validity of the knowledge databases involved, the technical requirements, the users' support and the interoperability.

The evaluation of the system can be conducted during its development [4], in accordance with iterative cycles, which are each characterized by an evaluation stage, the results of which will be used to improve the development on the next cycle, as well as a continuous improvement of the quality of the system.

This process consists of 4 phases [5]:

- Inception: for the analysis of needs, the general architecture of the system and the study of feasibility.

- Elaboration: helps to specify the use cases, to design the architecture of the system and to determine the reference architecture.

- Construction: during which the software is built using numerous iterations and many versions of the system.

- Transition: to hand the system over with its implementation to the end users and to train and support them for the intended use.

The evaluation will be made only at the stages of the last three phases:

- The evaluation of the development phase consists in checking whether the system to be designed and its architecture is helpful to the user.

- As for the construction phase, the evaluation consists in checking whether the designed model meets the user's requirements.

- Whereas the evaluation transition phase consists in testing the system carried out to identify the anomalies.

\subsection{Evaluation of the MDSS Impact on the Quality} and Safety of Care

Quality of care is the level reached by health organizations in terms of increasing the probability of expected results for individuals and populations in compatibility with the current state of knowledge.

The evaluation of the latter is an approach which must ensure that each patient is given the combination of diagnostic and therapeutic procedures that will ensure the best result in terms of health, in accordance with the current state of medical science, the cost for the same result, the least iatrogenic risk and for its greater satisfaction in terms of procedures, the results and the human contacts within the healthcare system.

Methods for evaluating the impact of MDSSs on the quality and safety of care vary according to the interests of the groups relevant to these systems (whether users, developers, patients, institutions or funders) and subjective or objective approach to the design of the study [6, 7].

\subsubsection{Objective Approach}

The first method: it is the only one used, for a long time, by research teams in medical informatics. It consists of comparison in a laboratory of the system's notifications to a standard established on the basis of experience (recommendations of practice or experts' opinions).

It is still insufficient to prove the clinical effectiveness of MDSSs, yet it is essential for their evaluation before being used in real practice. The 
second method: it is a clinical research method. It is the most commonly used approach today. It is based on the measurement of result variables, in studies where the independent explanatory variable is represented by the use or non-use of MDSS. In this case, we will quantify the impact of the use of MDSS on the performance of the clinician/MDSS system in terms of the benefits and risks associated with care with the control of some biases (the Hawthorne effect ...). Accordingly, objective evaluation measures the performance of an MDSS:

- either by comparing the system's notifications with a standard (expert's opinion or practice recommendations),

- or by measuring the impact of the MDSS:

(1) in terms of process variables (support to practice recommendations, etc.),

(2) in terms of health results or effects (decrease of side effects treatment, decrease in mortality, etc.).

Objective evaluation is based on:

- conducting controlled studies (randomized if possible),

- within the real practice of clinicians,

- practices after the expansion of DSSs.

To measure the MDSS impact on the safety and quality of practices and health results, the objective approach has now become the rule for evaluating the clinical impact of MDSS despite its limitations.

\subsubsection{A Subjective Approach}

After having been largely neglected until the end of the nineties, and faced with the need to better understand the factors of failure and success of the implementation of clinical information systems, the subjective approach has since then been subject to setting up dedicated structures in North America and Europe. In this approach, the human, the technical or the socio-cultural factors are taken into account as they are key decisive for the success or failure of the deployment of clinical information systems and may cause differences in impact between evaluating studies, in different contexts and in similar systems. The qualitative evaluation of these sociotechnical aspects of the MDSSs deployment is done thanks to a set of techniques available today, such as the observation of users while performing their activities, surveys, interviews, analysis of documents etc. [8, 9].

\subsection{Evaluation of the MDSS Impact on the Processes of Clinical Work and the Organization of Care}

In addition to the proved benefits of quality and safety of care, these systems can facilitate the work of caregivers and increase their productivity. However, the time of the evaluation must be appropriately chosen because the impact of DSSs on organizational processes is often only observed after many months of use. The impact on work habits can be evaluated through qualitative methods (focus groups, interviews), quantitative (samples), or data automatically collected by recording systems such as communications between professionals and system records [5].

Through the quantitative method we can try to evaluate the average time necessary to perform some tasks or to undertake some activities. Self-registration allows the user himself to collect his own activity data. An analysis of video data is possible. As for qualitative data, data analysis may involve computerized content analysis methods.

The use of these systems is widely appreciated and is considered as significant aid because it allows [10]:

- access to reference knowledge,

- prescribe medications online and from any workstation, examination, or procedure,

- practitioners and caregivers to simultaneously consult all patients' data, prescriptions, and test results,

- to receive assistance as appropriate check-ups or protocols, a graphical presentation of the relevant clinical data and complete information on prescribing and administration of medicinal products,

- to reduce work interruptions to clarify unclear written requests, or to obtain information from colleagues,

- to reduce phone calls between caregivers. 
The use of these systems therefore has a positive impact on the time between prescriptions and the arrival of the results of imaging or laboratory examinations or the administration of medications.

These delays were reduced from $23 \%$ to $92 \%$ in published studies [5]. The requirement that prescribers use these systems and the ability to view results remotely also reduces unwritten prescribing rates. Evaluation of the impact of MDSSs on clinical work processes and care organization can be indicators that are: the average time needed to administer medications to patients following the implementation of a prescription support system, or the number of clarifications requested by nurses on pre- and post-implementation. When analyzing these elements, some biases must be taken into account such as the concomitant use of another aid system, the change in the profile of patients, or the change in the pool of prescribers.

\subsection{Evaluation of the Validity of Knowledge Databases}

To make good decisions, the knowledge represented in the system must be valid and correctly represented.

A DSS is composed of:

- A database of facts: which represents the data statement problem to be solved, in our case, the criteria of care. The facts are often qualitative data (color, quality, properties, etc.) or numerical values;

- A knowledge database: containing the knowledge and recommendations of a given clinical area in a format that can be interpreted by the inference engine;

- An inference engine: which corresponds to the computer module, based on the knowledge database and the database of facts, models the reasoning to produce the decision-making aid. The reasoning is based on logic principles.

So, to evaluate the knowledge involved in DSSs, the quality of the sources, the rigor of translation of knowledge from source to database, and finally the quality and validity of the recommendations must be evaluated.

\subsection{Evaluating the Quality of Knowledge Sources}

The scope of the knowledge contained in the database must be compatible with the intended use of the system. The context can also be the type of user as well as his level of experience. The quality of knowledge sources depends on:

- the quality of the good practice guides involved: it must be based on systematic reviews of the scientific literature, etc. [11];

- the levels of evidence of the recommendations used: it must be clear (levels 1 to 5) and well taken into account by the algorithms of the inference engine;

- it should be noted that there are recommendations for the evaluation of good practice guides [12];

- it should also be checked whether a procedure for the practical updating of knowledge and rules has been arranged as the reliability of knowledge depends on their continuous updating. This must also be evaluated.

\subsection{Evaluating the Translation Quality of Knowledge from Sources to the DSS}

To evaluate the quality of knowledge translation from the sources to the DSS, and as we know that some criteria determine this quality, then we must evaluate whether:

- There is a connection between the knowledge representation model and the chosen inference engine (we can take the example of an inference engine based on a Bayesian model that must function with a knowledge database expressing probabilities),

- There is no penalization for the performance of the system by the representation model,

- The use of common standards of knowledge representation has been made whenever possible [13],

- There is a compatibility between the knowledge presentation format and the pre-existing systems in the DSS environment.

\subsection{Evaluating the Quality and Safety of the} Recommendations Provided

To evaluate the quality and safety of the 
recommendations provided, there are two cases:

(1) In the presence of a Gold Standard [14]:

- We will compare the recommendations given to a reference test (the Gold Standard) on a series of test cases,

- It appears that the effectiveness of some DSSs is not reflected in their performance relative to a Gold Standard.

(2) In the absence of a Gold Standard, this is often the case [15]:

- Instead of the Gold Standard, we will consider as a reference the experts' opinions or another system with the same ambitions,

- In this case the experts will be asked to note:

o The recommendations of the DSS,

o And those of experienced clinicians on specific issues.

- Obviously, this will be a blind study.

- When likely risks can be expected in the use of DSSs, an evaluation must ensure that they are as low as possible. Risk prediction is a notion to be included in the development cycle of the DSS [16].

\subsection{Evaluating the Technical Aspects}

The need to evaluate the system during its development stems from the fact that the results of the evaluation of each stage of the software development are used to improve development on the next cycle [17]. This results in a continuous improvement of the quality of the system. In this respect, ideally, the evaluation of decision support systems must be a continuous process strategically organized and not a one-off operation.

There are 3 types of technical requirements for an electronic system [18]:

- Non-functional requirements: safety, robustness, reliability, technical performance, ease of use and ease of maintenance or scalability,

- Functional requirements: the level at which the system can step back in a patient's past to give an opinion, etc.,
- The requirement related to the development process.

Some of the errors resulting from the use of DSSs were partly due to the technical aspects of the systems’ design [5].

\subsection{Evaluating the Users'Support}

To guarantee the success of a decision-making system, which is already very expensive, it is necessary to have the users' support. To achieve this, it is necessary to obtain the opinion of the latter throughout the system's life cycle, at the earliest stage possible, to allow a better adjustment of the development and implementation. This compendium will be done at the moment of the surveys through questionnaires, preferably validated, to favor the comparability of the various studies (the questionnaire of the end user, QUIS (questionnaire for user interaction satisfaction), TAM User Acceptance questionnaire, etc.) [19]. Video data can also be explored to provide information by analyzing the users' feedback to the system. There are also studies to compare the wishes expressed by users before implementation with their attitude towards the post-implementation DSS, which can also be called pre- and post-studies that are very significant. The evaluation of the users' support can be of interest both to the users' satisfaction and to the degree of use of the system. The factors determining this support would be the expected benefit of the system and simplicity of use [5].

\subsection{Evaluating Interoperability}

Interoperability is the ability of heterogeneous products and sets of computing products to work together. The use of common languages and protocols is one of the fundamental conditions for communication between computers and heterogeneous software, which gives the possibility of communication, program implementation or data transfer between different functional units so that the 
user has little or no need to know the characteristics of each unit. Multiple aspects can characterize this interoperability, namely the technical, communication and terminological aspects. Standards exist to ensure interoperability. As such, the HL7 enables the exchange of data between clinical systems. Semantic interoperability is ensured by standards: SNOMED, UMLS (unified medical language system), ICD (international classification of diseases), DRG (diagnosis related groups), CTV3 (clinical terms version 3).

The degree of interoperability is a criterion to be evaluated as the interoperability of a DSS with the basic information system on which it is based (example of the hospital information system) which is of crucial importance for the future of these systems [20].

To evaluate interoperability, it is necessary to consider the terminologies used, their compatibility with the pre-existing information system and the number of modules of the DSS complying with the interoperability standards provided that the DSS is dividable into modules.

\section{Ergonomic and Human Problems}

The work process of clinicians may be disrupted by ergonomic problems, including difficulties in accessing computers at the place and at the time of decisions, mostly in patients' rooms, poor adaptation of premises to the use of computers, difficulties in the use of poor quality of the users' interfaces (inconsistencies in presentation, poor grouping of on-screen items, overly complicated and cluttered screens, etc.), or lack of security procedures to avoid selecting the wrong patient or enter incorrect data. All these problems are the cause of difficulties in training and adaptation of users and of time losses compared to paper procedures [21].

It should also be noted that to gain the clinicians' trust and get them to accept and use the MDSS can be challenging tasks. The acceptance and use of MDSSs is initially weak especially if practitioners have not been engaged in the project from the beginning and the project objectives were not aligned with those of the healthcare facility [22, 23]. For instance, while design and work process barriers are often mentioned as the main barriers, the lack of trust of clinicians in the value of practice recommendations or in the reliability of the MDSSs often appear to be the first barriers to their use. This lack of confidence appears to be related to the lack of training of clinicians in the use of clinical information systems, but also to the existence or otherwise of a quality culture in the organization that must be considered as prerequisite for the decision to implement an MDSS.

Clinicians may also lack motivation to use MDSS because they feel they already know and follow the recommendations and that these recommendations are incomplete or that they do not apply to their patients. They often think that patients and work processes are different in their healthcare facility. It also appears that the quality and scope of MDDSs are not always sufficiently accurate or sufficiently broad for clinicians to participate in system notifications. This is especially true in the case of multiple pathologies for which there is no evidence of high level and whose recommendations for management can only be the subject of expert notification [24]. In this respect, many practitioners are unaware or do not participate in the recommendations of the system, and many of them receive data in the clinical information system only after consultation with the patient, which considerably reduces the MDSS potential benefit as they are not set off at the time and place of the consultation.

Therefore, it is essential that opinion leaders engage and communicate on the benefits of DSS; that clinicians are able to participate in the project design as early as possible; that work processes are analyzed, evaluated and, if necessary, redefined; that the content of knowledge bases are documented and based on recommendations with a high level of evidence; and that the necessary improvements are made to the 
design of the MDSS interventions so that their integration in the working process does not take place at the expense of its fluidity.

\section{Health Policy Makers and Need for Additional Work}

Promoting interoperability objectives and developing the knowledge databases necessary for the development of MDSSs is based on the development of standards for clinical practice guidelines. Moreover, incentives by health insurers and public authorities are likely to promote the installation, implementation and use of these systems. As such, health policy-makers should continuously review ways to promote the adoption of quality practices, including quality-based remuneration, and the provision of comparative data on their practices to clinicians, to realize the potential of clinical information systems and MDSS, and to improve the quality, safety and efficiency of care.

To enhance the design and implementation of MDSSs, further efforts are needed, namely the creation of more specific and binding implementation guides to promote the consistent and homogeneous application of standards; to improve knowledge about the effectiveness of different types of MDSS interventions on the quality of health practices and results; a better understanding of the factors that may facilitate the acceptability of MDSS interventions and their use by clinicians; a continuous assessment of the evolution of the systems, their use and their impact on the performance of clinicians and the improvement of the SADM accordingly [23].

\section{Conclusion}

Medicine is a scientific discipline but also a discipline of action that often requires decision-making. Several categories of computer systems can participate in various phases of this process including DSS. Theoretically, they improve the quality of care, but their practical integration is still far from being a reality for most health professionals. The close inter-relationship between recommendations, practitioner resources and local practices requires an accurate assessment of the impact of MDSSs to limit the negative consequences for patients. Therefore, the evaluation of DSS is a complex issue, which must benefit from a broad vision through the many areas to be considered, namely the clinical impact, impact on professional practices, validity of the knowledge represented, technical requirements, user support and level of interoperability. The evaluation of a decision-support system must not be reduced to a one-time task, it is a permanent process that begins at the design stage of the systems, hence the need for an overlap between the evaluation process and the development cycle of the system. Better consideration and better implementation of the methodological approach of this evaluation seem to be major criteria on which the success and future of DSS depend.

\section{References}

[1] Beck, J. R. 2011. "Medical Decision Making: 20 Years of Advancing the Field.” Med Decis Making 21 (1): 73-5.

[2] Wyatt, J., and Spiegelhalter, D.. 1991. "Field Trials of Medical Decision-Aids: Potential Problems and Solutions.” In Proc Annu Symp Comput Appl Med Care, 3-7.

[3] Ammenwerth, E., Brender, J., and Nykanen, P. 2004. "Visions and Strategies to Improve Evaluation of Health Information Systems.” Presented at the Reflections and Lessons Based on the HIS-EVAL Workshop in Innsbruck. Int J Med Inform. 73 (6): 479-91.

[4] Aubain, D. B. Méthodologies d'évaluation des systèmes d'aide à la décision.

[5] Jacobson, I., and Rumbaugh, G. Booch et J. 1999. Le processus Unifie de Developpement logiciel. Eds Eyrolles, Paris.

[6] Wyatt, J. 1997. "Quantitative Evaluation of Clinical Software, Exemplified by Decision Support Systems.” Int J Med Inform 47 (3): 165-73.

[7] Friedman, C.P., and Wyatt, J. C. 2001. "Publication Bias in Medical Informatics.” J Am Med Inform Assoc. 8 (2): 189-91.

[8] Beuscart-Zephir, M. C., Elkin, P., and Pelayo, S. 2007. "The Human Factors Engineering Approach to Biomedical Informatics Projects: State of the Art, Results, Benefits and Challenges.” Yearb Med Inform.:109-27. 
[9] Weir, C. R., Nebeker, J. J., and Hicken, B. L. 2007. “A Cognitive Task Analysis of Information Management Strategies in a Computerized Provider Order Entry Environment.” J Am Med Inform Assoc. 14 (1): 65-75.

[10] Lagouarde, P., Renaud-Salis, J. L., and Darmoni, S. 2010. "Etude des systèmes d'aide à la décision médicale" [Online]. Available: http://www.hassante.fr/portail/upload/docs/application/pd f/2011-01/etude_sadm_etat_des_lieux_2.pdf. [Accessed: 17-Feb-2016].

[11] Shiffman, R. N., Liaw, Y., and Brandt, C. A. 1999. "Computer-Based Guideline Implementation Systems: A Systematic Review of Functionality and Effectiveness.” $J$ Am Med Inform Assoc. 6 (2): 104-14.

[12] Alonso, P., and Bonfill, X. 2007. "Clinical Practice Guidelines: Elaboration, Implementation and Evaluation.” Radiologia 49 (1): 19-22.

[13] Boxwala, A. A., Peleg, M., and Tu, S. 2004. "GLIF3: A Representation Format for Sharable Computer-Interpretable Clinical Practice Guidelines.” $J$ Biomed Inform. 37 (3): 147-61.

[14] Berner, E. S. 2003. "Diagnostic Decision Support Systems: How to Determine the Gold Standard." J Am Med Inform Assoc. 10 (6): 608-10.

[15] Degoulet, P., and Fieschi, M. 1998. Informatique Médicale. Paris: Masson.

[16] Fox, J., and Thomson, R. 2002. "Clinical Decision Support Systems: A Discussion of Quality, Safety and Legal Liability Issues.” In Proc AMIA Symp., 265-9.

[17] Clarke, K., O’Moore, R., and Smeets, R. 1994. "A Methodology for Evaluation of Knowledge-Based Systems in Medicine.” Artif Intell Med. 6 (2): 107-21.
[18] Dorfman. 1997. Software Requirements Engineering, 2nd ed.. IEEE Computer Society Press.

[19] Ammenwerth, E., Mansmann, U., and Iller, C. 2003. "Factors Affecting and Affected by User Acceptance of Computer-Based Nursing Documentation: Results of a Two-Year Study.” J Am Med Inform Assoc. 10 (1): 69-84.

[20] Liaw, S. T., and Schattner, P. 2003. "Electronic Decision Support in General Practice.” What's the hold up? Aust Fam Physician 32 (11): 941-4.

[21] Bates, D. W., Cohen, M., and Leape, L. L. 2001. "Reducing the Frequency of Errors in Medicine Using Information Technology.” J Am Med Inform Assoc. 8 (4): 299-308.

[22] Sittig, D. F., Wright, A., and Ash, J. S. 2009. “A Set of Preliminary Standards Recommended for Achieving a National Repository of Clinical Decision Support Interventions.” In AMIA Annu Symp Proc., 614-8.

[23] Das, M., and Eichner, J. 2010. Challenges and Barriers to Clinical Decision Support (CDS) Design and Implementation Experienced in the Agency for Healthcare Research and Quality CDS Demonstrations (Prepared for the AHRQ National Resource Center for Health Information Technology under Contract No. 290-04-0016.) AHRQ Publication No. 10-0064-EF. Rockville, MD: Agency for Healthcare Research and Quality.

[24] Seroussi, B., Bouaud, J., and Denke, D. L. 2009. "Using Knowledge Modeling to Measure How Clinical Practice could Actually Be Evidence-based: A Preliminary Analysis with Arterial Hypertension Management.” Stud Health Technol Inform. 150: 668-72. 